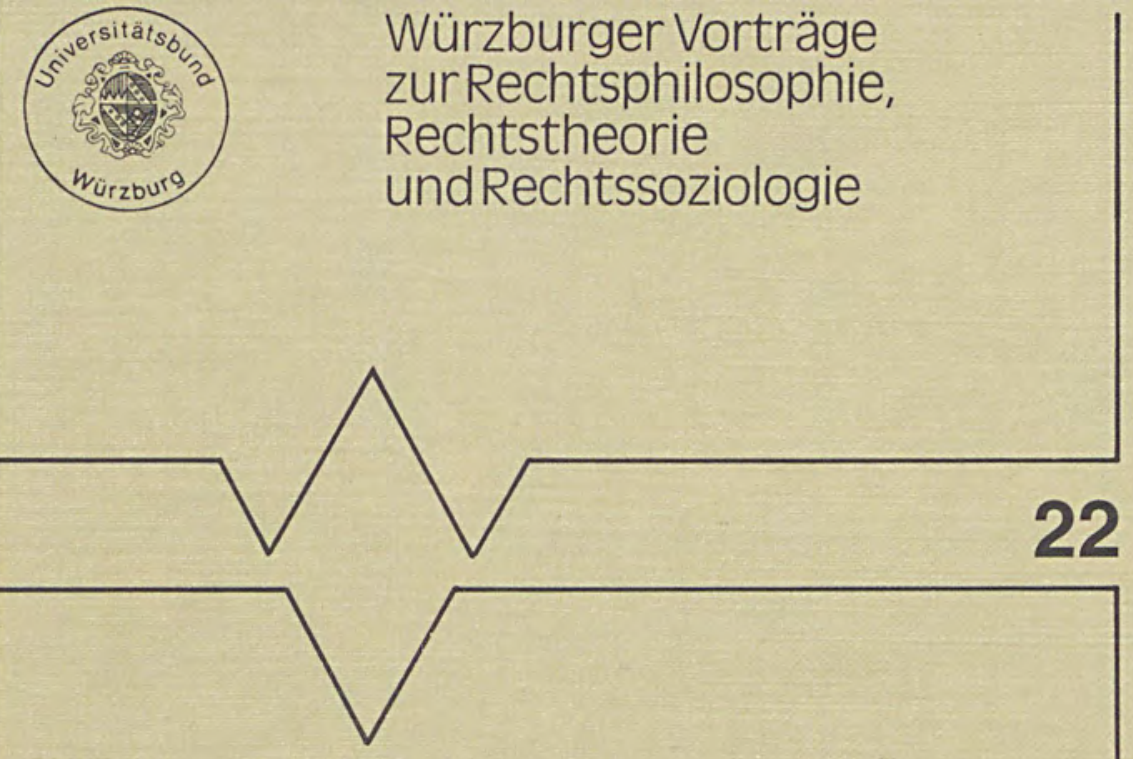

Michael Stolleis

Rechtsgeschichte als Kunstprodukt

Zur Entbehrlichkeit von „Begriff" und „Tatsache“

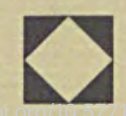

Nơmôs Verlag 


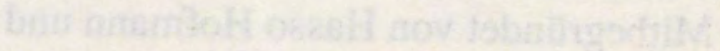

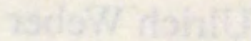


Würzburger Vorträge zur Rechtsphilosophie, Rechtstheorie und Rechtssoziologie

Herausgegeben von Horst Dreier, Edgar Michael Wenz und Dietmar Willoweit

Mitbegründet von Hasso Hofmann und Ulrich Weber

Heft 22 
Prof. Dr. Michael Stolleis

Rechtsgeschichte als Kunstprodukt

Zur Entbehrlichkeit von

„Begriff“ und „Tatsache“

Nomos Verlagsgesellschaft

Baden-Baden 
Vortrag gehalten am 10. Juli 1997

Die Deutsche Bibliothek - CIP-Einheitsaufnahme

\section{Stolleis, Michael:}

Rechtsgeschichte als Kunstprodukt : Zur Entbehrlichkeit von „Begriff“ und „Tatsache“ ; [Vortrag gehalten am 10. Juli 1997 / Michael Stolleis. - 1. Aufl. Baden-Baden : Nomos Verl.-Ges., 1997.

(Würzburger Vorträge zur Rechtsphilosophie, Rechtstheorie und Rechtssoziologie; H. 22) ISBN 3-7890-5098-9

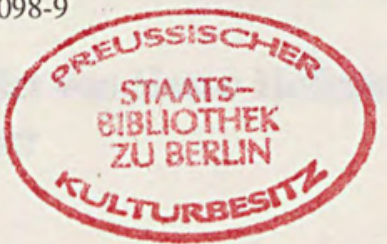

1. Auflage 1997

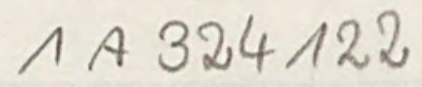

(c) Nomos Verlagsgesellschaft, Baden-Baden 1997. Printed in Germany. Alle Rechte, auch die des Nachdrucks von Auszügen, der photomechanischen Wiedergabe und der Übersetzung, vorbehalten. Gedruckt auf alterungsbeständigem Papier. 


\title{
Rechtsgeschichte als Kunstprodukt
}

\author{
Zur Entbehrlichkeit von »Begriff « und »Tatsache»
}

I.

Über Ideen und Begriffe, Worte und Tatsachen in der Historiographie ist in den vergangenen Jahrzehnten unübersehbar viel geschrieben worden. "Geschichte schreiben in der Postmoderne $\ll^{1}$ erlebt gegenwärtig offenbar einen Pendelschlag von angestrengter Verobjektivierung mit PC-gestützter Erfassung saller« Daten hin zur Wiederentdeckung der Phantasie und des Erzählens. Die Grenzen zwischen einem Werk der Geschichtswissenschaft, einem Roman und einem per »Einfühlung « und »dichter Beschreibung" gegebenen Kulturreport lösen sich auf. Und die Literarisierung von Wissenschaften, die ihr Objektivitätsideal nicht mehr verteidigen, macht auch vor der Rechtswissenschaft nicht halt. »Law and Literature« ist eine ausgebreitete intellektuelle Bewegung geworden ${ }^{2}$. Hayden White, Carlo Ginzburg, Stephen Greenblatt, Quentin Skinner und Clifford Geerds schwenken die Fähnlein der verschiedenen Richtungen. Die einen suchen die historische Wahrheit in Tiefenbohrungen in außergewöhnlichen oder entlegenen Quellenbeständen ${ }^{3}$, wieder andere rekonstruieren den kollektiven Kontext des Sprechens im

1 Chr. Conrad-M. Kessel (Hrsg.), Geschichte schreiben in der Postmoderne. Beiträge zur aktuellen Diskussion, Stuttgart 1994.

2 R. A. Posner, Law and Literature, Cambridge/Ms. - London 1988; K. Lüderssen, Produktive Spiegelungen. Recht und Kriminalität in der Literatur, Frankfurt a.M. 1991.

3 C. Ginzburg, Il formaggio e i vermi. Il cosmo di un mugnaio del '500, Torino 1976; H. Medick, A. Th. van Deursen, Graf. Ein Dorf aus dem 17. Jahrhundert, Göttingen 1997; H. Medick, Weben und Überleben in Laichingen, 1650-1900, Lokalgeschichte als Allgemeine Geschichte, Göttingen 1996. 
sozialen Raum, oder sie sammeln möglichst viele Daten über vergangene Gesellschaften und bringen sie durch intelligente Verknüpfungen zum Sprechen. Jeder von ihnen aber zweifelt mehr oder weniger laut an der Wissenschaftlichkeit seines eigenen Textes. Jeder beobachtet bei sich selbst mindestens Spurenelemente schöpferischer Willkür des Künstlers, der seinen Stoff arrangiert. Jeder Historiker, der die Stimmen der Toten hören und wiedergeben will, kann dazu nur seine eigene Stimme benutzen. Jeder weiß, daß es »die « historische Wahrheit nicht gibt, so wie es bei jeder »Beziehungsgeschichte « zwei, drei oder vier Wahrheiten gibt, die parallel zu erzählen wären.

Diese etwas kryptischen Bemerkungen mögen auf eine unkomplizierte Weise zu den Fragen der politischen Ideengeschichte und der Rechtsgeschichte führen, die Gegenstand dieses Vortrags sind. Diese Fragen kennen natürlich keine nationalen Begrenzungen. Dennoch gewinnt man öfter den Eindruck, als gebe es - jedenfalls in der Rechtsgeschichte - relativ abgegrenzte europäische Provinzen. Die methodische Debatte der Geschichtsschreibung in Europa und USA ist nur scheinbar konsistent. Sie wird vielmehr so geführt, daß viele der Diskutierenden die Werke der übrigen Diskussionsteilnehmer nur dem Namen nach kennen. Zwar wird manches übersetzt, aber es ist doch eine ziemlich gut belegbare Beobachtung, daß die anglo-amerikanische Debatte fast ganz ohne Kenntnis deutscher Texte auskommt, daß die französische Debatte weitgehend in sich geschlossen ist, während man sich in Italien, Spanien und Deutschland offenbar für das englische oder das französische »Zitatgebiet « entscheidet. Damit bin ich fast schon beim Thema. Nicht nur die Sprachen sind unterschiedlich, auch die jeweiligen historischen und intellektuellen Kontexte, innerhalb derer die Sprachen gebraucht werden. England und Frankreich, Italien und Deutschland (um nur diese vier zu nennen) haben den Übergang vom 19. in das 20. Jahrhundert sehr unterschiedlich erlebt, beschrieben und durch Beschreibung »begriffen«. Selbstverständlich führte die unterschiedliche Optik auf unterschiedliche Na- 
tionalgeschichten zu verschiedenen Standpunkten, diesseits und jenseits des Kanals, diesseits und jenseits des Rheins, diesseits und jenseits der Alpen oder der Pyrenäen. Der Übergang in das Industriezeitalter verlief zeitlich versetzt und unterschiedlich intensiv von der Mitte des 18. bis an das Ende des 19. Jahrhunderts. Und schließlich: auch die gedanklichen Traditionen, die dominanten philosophischen Schulen und der intellektuelle Habitus waren und sind sehr verschieden - auch dies unter den Europäern eine stete Quelle möglicher Mißverständnisse. Nur eine oberflächliche Europa-Rhetorik wird dies leugnen.

In Deutschland setzte in der Mitte des 19. Jahrhunderts eine erste Distanzierung vom klassischen Idealismus Hegels ein. Der wissenschaftliche Positivismus drängte nach vorne, die Naturwissenschaften entwickelten das prägende Paradigma. Aber schon am Ende des 19. Jahrhunderts zeigte sich eine erneute Gegenibewegung, die Kritik am Fortschritt, der Kulturpessimismus und die Katastrophenstimmung des Fin de siècle. Aus den Bruchstücken der ehemals relativ geschlossenen Systeme des Idealismus wuchsen jetzt diverse Neo-Philosophien, der Neokantianismus sowie seit dem ersten Weltkrieg der Neo-Hegelianismus samt Renaissancen von Fichte und Schelling. Unübersehbar gab es einen starken Zug zu neuer »Metaphysik«, eine Suche nach den Grundlagen bei den Vorsokratikern, einen heroischen Nihilismus und eine entsprechende Blüte von Sekten und Heilslehren aller Art.

Notwendig förderte diese Richtung wieder ihre Antipoden: eine Verschärfung des positivistischen Standpunkts in der modernen Logik, Wissenschaftstheorie und Sprachphilosophie (Ernst Mach, Moritz Schlick, Rudolf Carnap, Ludwig Wittgenstein) und in der Rechtswissenschaft die sog. Wiener Schule unter Führung von Hans Kelsen.

Auf diesem von Gegensätzen zerrissenen Untergrund spaltete sich auch die Methologie der Geschichtswissenschaften. Die herrschende Richtung der zwanziger Jahre pflegte die klassische politische Geschichte sowie die »Ideengeschichte«, wäh- 
rend Sozial- und Wirtschaftsgeschichte zurückgedrängt wurden. »Ideengeschichte« dieser Art ruhte auf letztlich platonischen philosophischen Überzeugungen vom Wirken ewiger Ideen oder jedenfalls anthropologischer Grundkonstanten in der Geschichte, von ihrer zeitweisen Verdunkelung und ihrem immer wieder siegreichen Hervorbrechen. Friedrich Meinecke hat dies am vermeintlich ewigen Antagonismus von Kratos und Ethos zu zeigen versucht ${ }^{4}$.

Die Rechtsgeschichte war diesem Muster ebenfalls verpflichtet, und zwar in ihrem nationalen Zweig (Deutsche Rechtsgeschichte) stärker als in der international vernetzten Forschung zur Geschichte des römischen Rechts, die sich in jenen Jahrzehnten zur Antiken Rechtsgeschichte ausweitete (L. Mitteis, L. Wenger). Besonders deutlich hat dies Heinrich Mitteis in seinen Publikationen nach 1945 formuliert. Für ihn bestand nach der Erfahrung des NS-Regimes die Aufgabe der Rechtsgeschichte darin, im Material der Quellen die leuchtenden Ideen der Freiheit und der Gerechtigkeit zu entdecken ${ }^{5}$. Auf der Grundlage eines mehr oder weniger von Hegel inspirierten Gedankengebäudes vermittelte diese Sicht auf eine - trotz aller Weltkatastrophen - immer wieder aufleuchtende positive Idee eine psychische Stütze. Die Rechtshistoriker sollten wissen, was sie tun, sollten in der Erforschung der Quellen einen »Sinn « sehen. Das Bekenntnisbuch von Heinrich Mitteis trug deshalb auch den Titel »Vom Lebenswert der Rechtsgeschichte«. Man muß diesen Titel normativ lesen: Die Rechtsgeschichte muß ein positives, ideales Telos haben! In diesem Sinn stellten sich die in gefährliche Nähe zum Nationalsozialismus geratenen »Germanisten ${ }^{6}$

4 M. Stolleis, Friedrich Meinecke's »Die Idee der Staatsräson« und die neuere Forschung, in: ders., Staat und Staatsräson in der frühen Neuzeit, Frankfurt a.M. 1990, $134 \mathrm{ff}$.

5 S. Gagnér, Zur Methodik neuerer rechtsgeschichtlicher Untersuchungen I. Eine Bestandsaufnahme aus den sechziger Jahren, Ebelsbach 1983, 9 ff.

6 J. Rückert - D. Willoweit (Hrsg.), Die Deutsche Rechtsgeschichte in der NS-Zeit, ihre Vorgeschichte und ihre Nachwirkungen, Tübingen 1995. 
wieder in den Dienst des demokratischen Staates und wiesen betont auf die klassischen Werte der Freiheit, der Gerechtigkeit und der sozialen Verantwortung hin.

Werfen wir einen Blick auf die durch den Nationalsozialismus schwer dezimierte und sich mühsam wieder regenerierende Geschichte des römischen Rechts, dann sehen wir dort mehrere Richtungen in Parallele: Einen Neohumanismus, der sich - vom geltenden Recht emanzipiert - der Textforschung verschrieben hatte und enge Beziehungen zu den übrigen Altertumswissenschaften hielt ${ }^{7}$. Daneben einen Neopandektismus, der gerade umgekehrt darauf pochte, das Studium des römischen Rechts könne nur in lebendiger Wechselbeziehung mit dem geltenden (Zivil-)Recht erhalten werden. Diese zweite Richtung war auch überzeugt, daß das römische Recht mit seiner jahrhundertelangen Durchbildung, mit seinem Formenreichtum und seinem Gehalt an common sense (ratio scripta) für die Dogmatik des geltenden Rechts die eigentliche Grundlage biete. Aus der Geschichte der Dogmen, hinter denen man im Prinzip gleiche, wenn auch in historischen Kontexten veränderte Gedanken vermutete, könne das aktuelle Recht »lernen«. In dieser Weise hat sich etwa pointiert Paul Koschaker geäußert ${ }^{8}$.

Die germanistische und die romanistische Richtung der Rechtsgeschichte konnte auf diese Weise von verborgenen idealistischen Prämissen zehren. Beide beriefen sich auf »ewige Werte«, »Naturrecht«, auf eine dem römischen Recht immanente Vernunft oder auf einen historisch wandelbaren, aber doch mit einem festen Kern versehenen Formenschatz tradierter Jurisprudenz.

Methodisch wurde dies wenig reflektiert. Erst in den sechziger Jahren, als die gesellschaftliche Unruhe auf die Universitäten erfaßte, sahen sich die Rechtshistoriker plötzlich mit

7 F. Wieacker, Römische Rechtsgeschichte, 1. Abschn., München 1988, § 3, insbes.

S. $46 \mathrm{ff}$. zum »Entdeckungszeitalter der neueren Romanistik «.

8 P. Koschaker, Europa und das römische Recht, München 1947 u.ö. 
Forderungen nach einer materialistischen Rechtsgeschichte konfrontiert ${ }^{9}$, die ihrerseits wiederum aus dem Fundus des 19. Jahrhunderts lebte und keineswegs in der Lage war, ihre eigenen Postulate auch nur ansatzweise umzusetzen. Gleichzeitig war auf breiter Front eine Rezeption der analytischen Philosophie zu beobachten ${ }^{10}$.

Interessanterweise häufen sich die Wandlungen um das Jahr 1967. In Erich Rothackers »Archiv für Begriffsgeschichte« wurden 1967 nebeneinander zwei große Projekte angekündigt: Das von Joachim Ritter herausgegebene »Historische Wörterbuch der Philosophie ${ }^{11}$ und das Werk »Geschichtliche Grundbegriffe« von Otto Brunner, Werner Conze und Reinhart Koselleck $^{12}$. Das philosophische Projekt Ritters grenzte sich zwar gegen die auf Descartes zurückgehende Position ab, es könnten durch hinreichende philosophische Begriffsklärung eine endgültige Bereinigung der begrifflichen Verwirrung und die Etablierung einer »science universelle« erreicht werden. Wäre dieses Ufer der Klarheit erreicht, könnte man die verworrene und

9 U. Wesel, Zur Methode der Rechtsgeschichte, Krit. Justiz 1974, 337-368; kritisch J. Rückert, Zur Erkenntnisproblematik materialistischer Positionen in der rechtshistorischen Methodendiskussion, in: Zeitschrift für Historische Forschung 5 (1978), 257-292. Zusammenfassend $P$. Landau, Bemerkungen zur Methode der Rechtsgeschichte, in: Zeitschrift für Neuere Rechtsgeschichte 1980, 117-131; M. Senn, Rechtshistorisches Selbstverständnis im Wandel, Zürich 1982.

10 Prägnante Summen bei D. Simon, Rechtsgeschichte, in: A. Görlitz (Hrsg.), Handlexikon zur Rechtswissenschaft, München 1972 (Taschenb. Reinbek 1974, 314-318); F. Wieacker, Methode der Rechtsgeschichte, in: Erler - Kaufmann (Hrsg.), Handwörterbuch zur Deutschen Rechtsgeschichte (HRG), Bd.III (1984), Sp. 518-526.

11 Als Neubearbeitung von Rudolf Eisler, Wörterbuch der philosophischen Begriffe, 1897, 4. Aufl. 1927.

12 J. Ritter, Leitgedanken und Grundsätze des Historischen Wörterbuchs der Philosophie, in: Archiv für Begriffsgeschichte 1967, 75-80; R. Koselleck, Richtlinien für das Lexikon politisch-sozialer Begriffe der Neuzeit, aaO. 81-99. Vgl. auch $R$. Koselleck (Hrsg.), Historische Semantik und Begriffsgeschichte, Stuttgart 1979. - Der heutige Diskussionsstand bei Melvin Richter, Appreciating a Contemporary Classic: The Geschichtliche Grundbegriffe and Future Scholarship, in: H. Lehmann - M. Richter (ed.), The Meaning of Historical Terms and Concepts. New Studies on Begriffsgeschichte, German Historical Institute, Occasional Paper Nr.15, Washington 1996, $7 \mathrm{ff}$. 
dunkle Geschichte hinter sich lassen. Ritter wollte eine »Geschichte des Begriffs«, weil er Philosophie weniger als Suche nach festen Ergebnissen, sondern als Entfaltung eines historischen Prozesses sah (philosophia perennis). Er wollte damit die Mitte zwischen Geschichtslosigkeit und historischer Relativierung halten. Seine Fragestellung war allerdings klar philosophisch, nicht historisch.

Ebenfalls 1967 begannen die Arbeiten an dem Werk »Geschichtliche Grundbegriffe. Historisches Lexikon zur politischsozialen Sprache in Deutschland ${ }^{13}$. Es ist nunmehr, etwa fünfundzwanzig Jahre nach seinem Beginn, abgeschlossen worden. Anders als bei Ritter war seine Intention »historisch «. Das Lexikon sollte den Wandel des Sprachgebrauchs jener berühmten und folgenreichen Zeit des Übergangs zwischen 1750 und 1850 (Sattelzeit) beschreiben, vor allem in zentralen »Begriffen «. Ausgewählt wurden solche Begriffe, die für die Neuzeit »wichtig" schienen, andere wurden beiseitegeschoben. Nur politischsoziale "Bedeutungen" sollten von Interesse sein. Ziel der Beschreibung war es, durch Analyse der »Bedeutungen « eines kohärenten Wortfeldes die historische »Realität « zu erfassen. Verschiebungen der Bedeutung galten als Signale für eine Verschiebung in der Welt von Politik und Gesellschaft.

Freilich wußten die Herausgeber, insbesondere Koselleck, daß »Realität« nicht pur zu haben war. Nur was »begriffen« wird, ist Geschichte. Deshalb unterschied man Wort und Begriff. Worte sollten eindeutig sein oder jedenfalls exakt definiert werden können, Begriffe galten als mehrdeutig und interpretierbar. Begriffe sollten als reichere Konzentrate von Wortbedeutungen über den Worten stehen, sollten gewissermaßen wie reichbeladene Schiffe eine Fracht unterschiedlicher Bedeutungen transportieren.

So sehr ich mit den Zielvorstellungen einer historischen Begriffsgeschichte in diesem Sinne übereinstimme, die Unterschei-

\section{Stuttgart $1972 \mathrm{ff}$.}


dung von Wort und Begriff scheint mir unhaltbar. Worte sind keineswegs eindeutig, wie alle Erfahrung lehrt, und die zu ihrer angeblich exakten Definition verwendeten Worte sind ebenfalls schwankende Gebilde. Eine »exakte Definition« gibt es nicht. Was wir erreichen können, ist allenfalls eine innerhalb bestimmter Kommunikationsgemeinschaften stabile Konvention des Verständnisses, ablesbar an den Reaktionen der Teilnehmer im Prozeß der Verständigung. Unschärfe und Sozialabhängigkeit der Sprache sind nicht zum Verschwinden zu bringen. Die sog. Begriffe sind im Sinne der Semiotik »Worte«, nicht mehr und nicht weniger. Das ist, um es deutlich zu sagen, eine nominalistische Position.

Hinter der im wissenschaftlichen Alltag (speziell Deutschlands) hartnäckig behaupteten Differenz zwischen Worten und Begriffen stecken vermutlich Restbestände von Begriffsrealismus, von Glauben an »Ideen « und »Wesenheiten «, kurzum: von Metaphysik. Die Worte »Begriff" und »Tatsache" sind im 18. Jahrhundert in die deutschen Fachsprachen der Philosophie und der Jurisprudenz eingeführt worden ${ }^{14}$. Christian Wolff, dem dies bezüglich des Wortes »Begriff« in die philosophische Fachsprache zugeschrieben wird, hat ganz anders als Koselleck das »Wort" für das ungenaue Element des Alltagssprachlichen gehalten, während er unter $» B$ egriffen $"$ gerade die gereinigten, präzise wissenschaftlichen, für die Klassifikation unterschiedlicher empirischer Erscheinungen geeigneten Worte verstand ${ }^{15}$. Letztlich läuft diese Differenz auf den alten, hier nicht zu erneuernden Streit zwischen einem objektiv-idealistischen und einem

14 H. Kiefner, Zur Sprache des Allgemeinen Landrechts, in: B. Dölemeyer - H. Mohnhaupt (Hrsg.), 200 Jahre Allgemeines Landrecht für die preußischen Staaten, Frankfurt a.M. 1995, 23 ff. (69 ff.), weist nach, daß die alte res facti in einer Übersetzung aus dem Englischen (matter of fact) erstmals 1756 im Deutschen bei Spalding auftaucht, im juristischen Bereich erstmals 1778.

15 W. Schneiders (Hrsg.), Christian Wolff 1679-1754. Interpretationen zu seiner Philosophie und deren Wirkung, Hamburg 1983, dort insbes. G. Ungehener, Sprache und symbolische Erkenntnis bei Wolff, $89 \mathrm{ff}$. 
nominalistischen Verständnis der Sprache und der Weltdeutung durch Sprache hinaus.

Aber unabhängig von den Differenzen im philosophischen Hintergrund hat der durch Koselleck eingeschlagene Weg eine Fülle neuer Ergebnisse gebracht. Man lernte, auf die SchlüsselWorte zu achten, die Analyse der Bedeutungen als Zugang zu dem Denken vergangener Zeiten zu verstehen. Die diskreditierte, philosophisch vom deutschen Idealismus inspirierte »Ideengeschichte ${ }^{16}$ schien plötzlich in erneuerter Gestalt wieder möglich zu sein, nämlich als eine eng an die Sozial-, Wirtschafts-, Politik- und Rechtsgeschichte angeschmiegte Begriffsoder Wortgeschichte. Man mußte annehmen, daß Verschiebungen der Realität zu einer Verschiebung der Sprache führten umgekehrt konnte man aber auch fragen, ob auch bewußt eingeführte und durchgesetzte Änderungen des Sprachgebrauchs die Realität umgestalten konnten. Wie auch immer - der Akzent lag auf »Geschichte " und auf der Vermeidung »normativer Implikationen ${ }^{17}$. Die Sprachanalyse sollte nicht die Probleme als Scheinprobleme entlarven, sondern zum genaueren Verständnis des Sprachgebrauchs einer Zeit, d.i. ihres Denkens, beitragen helfen.

Von der Wechselwirkung zwischen sprachlich gefasstem Denken und materieller Lage ging auch die ebenfalls seit 1967 geführte neomarxistische Debatte des Westens aus. Selbst wenn dort die Priorität der materiellen Basis behauptet wurde, so war doch stets klar, daß auch der vorauseilende Gedanke die Basis verändern konnte. Das oft beschworene »revolutionäre Bewußtsein « mußte, wenn es nicht ganz als passive Funktion der materiellen Bedingungen erscheinen sollte, eine gewisse aktive Autonomie besitzen. Anders wäre es auch nicht verständlich, daß gerade kommunistische Systeme erhebliche Anstrengungen zur

16 Zum Hauptvertreter Friedrich Meinecke siehe M. Erbe (Hrsg.), Friedrich Meinecke heute, Berlin 1981.

17 K. v. Beyme, Politische Ideengeschichte, Tübingen 1969, 46. 
Lenkung des Sprachgebrauchs unternahmen, also offenbar doch eine Steuerung der Realität über Sprache und Denken für möglich hielten ${ }^{18}$.

Nachdem ich meinen ersten Einwand angedeutet habe, was die Unterscheidung von Worten und Begriffen angeht, will ich auch meinen zweiten Einwand vorbringen: Er betrifft die Unterscheidung von Worten und Sachen (Tatsachen). Nimmt man an, da $ß$ »hinter « der verwirrend unklaren Sprache die harte historische Realität auf ihre Entdeckung wartet, dann zerfällt die Welt in eine sprachliche und eine nichtsprachliche Seite. Entsprechend hat man eine Dichotomie von Wortforschung und Sachforschung aufgebaut ${ }^{19}$.

Gewiß kann Sprache keine realen Berge versetzen, keine wirklichen Schlachten schlagen, keine Toten lebendig machen. Insofern gibt es (banalerweise) historische $»$ Fakten $«$ und es gibt, wenn sie versunken sind, ihre Spuren. Diese Spuren sind (für den Historiker wie für den Richter) »Beweise«, die durch Schlußfolgerungen verknüpft werden können und einen gewissen, innerhalb pragmatischer Grenzen ausreichenden Grad an Gewißheit ergeben. Alle diese Spuren bedürfen der Interpretation, deren theoretischer Rahmen so angelegt sein muß, daß die Spuren gedeutet, verstanden oder entschlüsselt werden können. Wie auch immer dies geschehen mag, es ist ein unentrinnbar an die Sprache gebundener Diskurs, der allerdings auf außertextuelle Realität bezogen sein muß. Nur in der Sprache kann Geschichte als geistiges Konstrukt erstehen; denn die Welt, die wir erfahren und begreifen, ist sprachlich konstituiert. Indem wir

18 B. Vierneisel, Das Erinnerungsarchiv. Lebenszeugnisse als Quellengruppe im Institut für Marxismus-Leninismus beim ZK der SED, in: M. Sabrow (Hrsg.), Verwaltete Vergangenheit. Geschichtskultur und Herrschaftslegitimation in der DDR, Berlin 1997, 117-144 belegt eindrucksvoll, wie die Aufzeichnungen systematisch an die Parteilinie angepaßt wurden.

19 T. Giaro, Echtheitsindizien. Eine Fallstudie zu verus, verius, verissimus, in: Orbis Iuris Romani. Journal of Ancient Law Studies, Masaryk University of Brno, I (1995) 78-109. 
einen historischen Sachverhalt formulieren, erschaffen wir ihn als selbstverantwortete Abbreviatur von Informationen und Interpretationen. Er existiert nur als Sprache und kann nur durch sie vermittelt werden. Geschichtsschreibung ist also nie direkter Zugang zu einer »hinter« der Sprache existierenden Realität. Sie ist sprachliche Aneignung von (nur) sprachlich vermittelbaren Botschaften. Wird über Vergangenes nicht mehr gesprochen, verschwindet es und zurück bleiben unbegriffene Steine und unverstandene Zeichen. Erst durch Benennung - durch den Taufakt, wenn die theologische Parallele nicht erneut verwirrt werden sie lebendig und kenntlich.

Wenn Geschichte als gesellschaftliche Konstruktion vergangener Wirklichkeit nur durch Sprache verständlich, ja wenn sie durch Sprache erst wirklich geschaffen wird, dann muß auch die Grenze zwischen Historiographie und Fiktion unscharf werden. Historiker und Dichter schöpfen aus dem Brunnen der Erinnerung und beide formen aus diesem Material mit dem Medium der Sprache einen imaginativen Text. Peter Weiss hat dies - in der Lage des Jahres 1945 - beispielhaft beschrieben: „Schon jetzt, drei Monate nach dem Ende des Kriegs, schienen viele unserer Erinnerungen zweifelhaft geworden zu sein, was wir festhalten wollten, war bereits von einem dichten Gewebe lügenhafter Geschichtsschreibung umhüllt, die Vorgänge standen im Begriff, zwischen Mythen unterzugehen, und es galt dies für beide Seiten, die unsre und die des Gegners. Welchen Gegenstand auch immer wir aufnehmen wollten, er war verunstaltet .... ${ }^{20}$. Geschichtsschreiber und Geschichtenerzähler sind Geschwister im Geiste. Ich kann dies nur konsequent, keineswegs skandalös finden, sehe aber, daß die Mehrzahl der Historiker auf diese Unterscheidung großen Wert legt, offenbar entweder in der Sorge,

20 P. Weiss, Ästhetik des Widerstands, Bd. 3, Frankfurt a.M. 1991, 151. Zitiert bei Vierneisel (Anm.18), 128. 
mit Schriftstellern verwechselt zu werden oder (seriöser) aus Sorge um den Wissenschaftscharakter des eigenen Tuns ${ }^{21}$.

Hat man sich aber erst zu der Einsicht durchgerungen, daß der Historiker nur eine gelehrte und sich auf ältere Texte und Zeichen stützende Spezies der Gattung »Dichter/Schriftsteller" ist, dann verliert der changierende Grenzbereich zwischen gesicherter Geschichtsschreibung und frei erfundener Dichtung seine Schrecken. Der Unterschied reduziert sich dann auf die Frage der Kontrollierbarkeit anhand von Quellen sowie auf das wissenschaftsethische Gebot wissenschaftlicher Redlichkeit, die Genres nicht in der Absicht der Täuschung zu vermischen. Im Ergebnis wird oft der Dichter der bessere Historiker sein, gelegentlich ist der Historiker der Zuverlässigere, der etwa zeigen kann, wie täuschend die subjektive Zeiterfahrung sein kann oder wie täuschend manchmal die Oberfläche der vermeintlichen Kausalitäten. Beide »erschaffen« eine Welt und beide verwenden ein Kriterium der »Wahrheit«, das sich von der sog. exakten Beweisbarkeit unterscheidet.

Eine jüngst geführte Debatte um die Rolle der »Phantasie « in der Geschichtsschreibung kann dies illustrieren ${ }^{22}$. Ein Mediävist warf dem anderen einen zu freien, zu phantasievollen Umgang mit den Quellen vor ${ }^{23}$. Der Angegriffene entgegnete, und ich meine zu Recht, schon die Quellen selbst seien Artefakte, sie seien ebenso subjektiv wie die Interpretationen späterer Historiker. Verstehen sei ein dialogischer Vorgang zwischen neuer In-

21 Clifford Geertz, Thick Description: Toward an Interpretive Theory of Culture, in: C. Geertz, The Interpretation of Cultures. Selected Essays, New York 1973 (dt. Dichte Beschreibung. Beiträge zum Verstehen kultureller Systeme, 3. Aufl., Frankfurt a.M. 1994).

22 J. Fried, Die Garde stirbt und ergibt sich nicht. Wissenschaft schafft die Welten, die sie erforscht: Das Beispiel der Geschichte, in: Frankfurter Allgemeine Zeitung v. 3.4.1996.

23 G. Althoff, Von Fakten zu Motiven. Johannes Frieds Beschreibung der Ursprünge Deutschlands, HZ 260 (1995) 107-117; J. Fried, Über das Schreiben von Geschichtswerken und Rezensionen, a.a.O. 119-130. 
formation und gespeichertem Wissen, gebunden an Sprache und zeitlichen und persönlichen Kontext, kurz: schöpferische Vorgänge, bei denen die Phantasie eine grundlegende, jedenfalls heuristische Rolle spiele. Diese Feststellungen sind keineswegs neu. $» \mathrm{Da}$ die Einbildungskraft ein notwendiges Ingredienz der Wahrnehmung selbst sei, daran hat wohl noch kein Psychologe gedacht «, notierte Kant ${ }^{24}$. Und wir bestätigen uns diese Beobachtung unaufhörlich: Kinder spielen »Stille Post « und amüsieren sich über die Verdrehungen, die eine ins Ohr gesagte Botschaft schon nach wenigen Stationen erleidet; ohne Kants »produktive Einbildungskraft« könnten sie gar nichts wahrnehmen. Erwachsene erschaffen sich aus dem Wust voyeuristischer Desinformationen der sog. Regenbogenpresse eine eigene Realität, die mehr über ihre Sehnsüchte besagt als über den Realitätsgehalt des Berichteten. Die Formeln »ich habe Sie wohl falsch verstanden«, »ich habe mich mißverständlich ausgedrückt" oder »meinen wir wirklich dieselbe Sache?« sind uns geläufig, weil wir uns ständig durch einen Wald von Mißverständnissen tasten und unsere Anstrengung der Orientierung darin besteht, alte und neue Information zu kombinieren und dabei die eigene Subjektivität und die der Kommunikationspartner einzukalkulieren, um das Schattenwesen zu ermitteln, das wir »Tatsache« zu nennen pflegen.

Die neuere Hirnforschung bestätigt das Mißtrauen gegenüber der Zuverlässigkeit des Erinnerten. Wir lernen aus ihr, daß der naheliegende Vergleich zwischen Gehirn und Computer mehr falsch als richtig ist. Die gespeicherten »Engramme « der Vergangenheit sammeln sich im Gedächtnis nicht wie auf einem Film oder im Computerprogramm. Unter der Schädeldecke befindet sich keine Festplatte, die ein exactes Replay ermöglicht. Vielmehr arbeitet das Gehirn offenbar viel kreativer, etwa wie

24 I. Kant, Kritik der reinen Vernunft (1781), Theorie-Werkausgabe in 12 Bände, Bd. 3, Frankfurt a.M. 1968, 176 Anm.*. 
ein Collagekünstler oder Regisseur. Es schafft die Erinnerungen um, es arrangiert sie neu ${ }^{25}$. Erinnerungen werden mit Phantasien vermischt und durch Wunschvorstellungen, Idiosynkrasien und Vorurteile umgestaltet. Erinnerungen werden verwischt und durch eigene Inhalt ergänzt oder verdrängt. Die neuen Informationen werden in die bereits gespeicherten »eingelagert", sie verändern also auch jene.

Das Gehirn sortiert die neuen Eindrücke innerhalb eines »Rahmens" gefestigter Vorstellungen. Von einer exakten Speicherung historischer Wahrheiten kann also nicht die Rede sein.

Und möglicherweise vollzieht sich bei der Wiederbelebung und sprachlichen Umsetzung des Erinnerten eine erneute Verformung. Die erstaunliche Unzuverlässigkeit bei der Speicherung und Wiedergabe ganz einfacher Vorgänge kann anhand von Experimenten zur Qualität strafprozessualer Zeugenaussagen gezeigt werden ${ }^{26}$. Erst recht zeigt sie sich bei jahrhundertelanger oraler Überlieferung, bei der jede Generation neue Details erfindet und als verbesserte oder aktualisierte Fassung an die nächste Generation weitergibt ${ }^{27}$.

Als 1492 »Indien« entdeckt wurde, war es Amerika, aber ohne Benennung war es eben noch nicht »Amerika«. Die Schreib- und Sprechakte über jene Entdeckung formten den

25 E. Winograd - U. Neisser (ed.), Affect and Accuracy in Recall, Cambridge University Press 1993; G. Roth - W. Prinz (Hrsg.), Kopfarbeit. Gehirnfunktionen und kognitive Leistungen, Heidelberg-Berlin-Oxford 1996; H. Meier - D. Ploog (Hrsg.), Der Mensch und sein Gehim. Die Folgen der Evolution, München-Zürich 1997.

26 Etwa dadurch, daß man eine auffällig gekleidete Person durch die Sitzbankreihen des Hörsaals stürmen, etwas rufen oder eine andere Person scheinbar attackieren läßt. Die Studierenden, die den (wenige Sekunden währenden) Vorgang beschreiben sollen, können sich regelmäßig noch nicht einmal über Zeitdauer, Geschlecht der Person, verwendete Worte u.ä. einigen (Frdl. Mitteilung meines Kollegen Herbert Jäger).

27 A. Heubeck, Die homerische Frage. Erträge der Forschung (Bd. 27), Darmstadt 1974, 130 ff.; J. Latacz, Homer. Tradition und Neuerung, Wege der Forschung (Bd. 463), Darmstadt 1979; U. Hölscher, Die Odyssee. Epos zwischen Märchen und Roman, München 1988, insbes. 35 ff. 
neuen Kontinent ${ }^{28}$. Sowohl die Niederschrift der ersten Eindrücke als auch die Rezeption der Berichte in der Alten Welt waren kreative Akte. Columbus schilderte die neuen Landschaften und die Menschen als Paraphrasen seiner Vorstellungen des Paradieses und er verwendete dabei die Topoi des locus amoenus der antiken Literatur ${ }^{29}$. In den Wäldern ließ er Nachtigallen schlagen, obwohl es im damaligen Mittelamerika nachweislich keine Nachtigallen gab ${ }^{30}$. Seine von Europa mitgebrachten Vorstellungen von Indien verbanden sich mit dem, was er sah. Nicht anders erging es den (fälschlich?) so genannten Indios. Sie interpretierten Pferde und Reiter als göttliche Mischwesen und deuteten die Feuerwaffen (in ihrem Kontext ganz schlüssig) als Zeichen überirdischer Kraft. Die Leser der Berichte in Europa schließlich erschufen sich eine »Neue Welt» aus einem Gemisch von Vorurteilen, Phantasien und sprachlich vermittelter sog. Realität. So sind die heutigen Forschungen über die Entdeckung der Neuen Welt vor allem Forschungen über die Bilder der Neuen Welt in den Köpfen der Europäer, wie gerade jüngst am Beispiel des Kannibalismus gezeigt wurde ${ }^{31}$.

Zur Vermeidung von Mißverständnissen sei zum Abschluß dieses ersten Teils betont: Natürlich gibt es Unterschiede zwischen Historiographie und Fiktion. Die Phantasie des Historikers soll durch methodische Postulate, wie sie seit dem 17. Jahrhundert akzeptiert sind, gezügelt sein ${ }^{32}$. Seine Hypothese soll je-

28 W. Reinhard (ed.), Il Mondo Nuovo nella coscienza italiana e tedesca del Cinquecento, Bologna 1992, Introduzione.

29 E. R. Curtius, Europäische Literatur und Lateinisches Mittelalter, 5. Aufl. Bern und München 1965, Kap. 10, $\$ 6$.

30 E. W. Palm, Spanien und die Neue Welt, in: ders., Heimkehr ins Exil. Schriften zu Literatur und Kunst, Köln - Weimar - Wien 1992, 1-54 (7: „Die neue Realität wird in den Sehformen des späten Mittelalters begriffen«); ders., Zwischen Paradies und Wirklichkeit. Bilder von der Neuen Welt, aaO. 413-423.

31 A. Menninger, Die Macht der Augenzeugen. Neue Welt und Kannibalen-Mythos, 1492-1600, Stuttgart 1995.

32 Widerspruchsfreiheit, Trennung von Bericht und Bewertung, Trennung zwischen Quelle und eigener Darstellung, Quellen- und Urkundenkritik zur Ermittlung der »besten« Quelle. 
derzeit zur Falsifizierbarkeit bereitstehen, wenn andere, »bessere « Zeugnisse auftauchen. Allgemeine Annahmen über »normales « menschliches Verhalten, bei denen die Zeitgebundenheit der Normalität nicht reflektiert wird, sind verdächtig. Erst recht gelten Behauptungen ohne Angabe von Quellen als »vorläufig" oder als unseriös.

Andererseits muß nicht alles neu bewiesen werden; nicht alles ist unsicher. Die Ökonomie der Kräfte verbietet es, alles zu bezweifeln. Solange Ereignisse ausreichend belegt sind, werden wir unsere Kräfte nicht darauf verschwenden, sie noch einmal sicherer zu beweisen. In diesem Sinne hat auch jener Mediävist, Johannes Fried, versichert, daß Karl d. Große wirklich gelebt habe, daß er ein »matter of fact « $\operatorname{sei}^{33}$. Ebenso wird man bis auf weiteres annehmen, daß Cäsar an den Iden des März ermordet wurde und daß Alexander 333 bei Issus eine Schlacht geschlagen hat (bzw. schlagen ließ). Man mag diese Konventionen des Unbezweifelbaren »Tatsachen" nennen, aber man sollte nicht vergessen, daß es geistige Konstrukte sind.

II.

Meine Position wird vielleicht deutlicher, wenn ich skizziere, welches Arbeitsfeld sich dem Historiker oder Rechtshistoriker eröffnet, der sich mit der $»$ Bedeutung der Wörter ${ }^{34}{ }^{4}$ beschäftigt. Meine eigenen Studien in der Frühen Neuzeit kreisen um die Fragen der Entstehung des modernen Staates, seiner Gesetzgebung und Verwaltung, um die seinen Aufstieg begleitende Wissenschaftsgeschichte des öffentlichen Rechts, um die theoretischen Begründungen des Staates, seines Verfassungs- und Ver-

33 J. Fried (Anm.22); H. Vollrath, Geschichtswissenschaft und Geschichtsschreibung. Zur Diskussion um das Buch »Der Weg in die Geschichte « von Johannes Fried, in: Zeitschr. f. Geschichtswissenschaft 43 (1995) 451-459.

34 Die Bedeutung der Wörter. Festschrift für Sten Gagnér zum 70. Geb., hrsgg.v. M. Stolleis u.a., München 1991. 
waltungsrechts. Ich möchte wissen, wie und warum es zur Entstehung eines von religiösen und moralischen Bindungen getrennten politischen Denkens kam. Wie und warum verwandelte sich der mittelalterliche Lehenstaat in den modernen absolutistischen Territorial- und Anstaltsstaat? Auf welchen Wegen begleitete die politische und juristische Theorie diesen Vorgang? Wie verwendete sie ihr aus der Antike, aus der mittelalterlichen Scholastik, aus Kirchenrecht und römischem Recht gespeistes Reservoir an $»$ Begriffen ${ }^{35}$ ?

Wenn wir hierzu Näheres wissen wollen, analysieren wir Texte jener Zeit. Diese Texte sind uns fremd, sie sind in einer Sprache geschrieben, die uns fern liegt. Wir studieren den Sprachgebrauch jener Zeit, wir versuchen zu ergründen, was bestimmte Worte »bedeuteten«. Andere Wege haben wir nicht. Wir formulieren historische Sachverhalte in Sprache, und wir gewinnen, was wir »historische Sachverhalte«, »Tatsachen« oder "facts" nennen, aus der Beobachtung, wie bestimmte Worte eingesetzt werden.

- Diese Perspektive, die sich zwar einerseits nicht aus dem Gefängnis der Gegenwart und ihren optischen und semantischen Verhexungen befreien kann, die aber andererseits der Brauchbarkeit anachronistischer Begrifflichkeit bei der Entschlüsselung und Rückübersetzung älterer Wortbedeutungen mißtrauisch gegenübersteht, hat gewisse Vorteile: Sie kann zum Beispiel den älteren Beschreibungsmustern sogenannter Kausalitäten zwischen Gedanke und Tat entkommen. Die Vorstellung, daß der Denker (zunächst folgenlos) denkt, und daß der Tatmensch (davon angeregt) dann das Gedachte ausführt, ist ein für die meisten Fälle zu einfaches und unbrauchbares Modell. Das gleiche gilt für den umgekehrten Vorgang, bei welcher der Tat der kommentierende und deutende Gedanke nachfolgt. In der Rechts- und Verfassungsgeschichte sind auf diese Weise der

35 A. Padoa Schioppa (ed.), Legislation and Justice, (The Origins of the Modern State in Europe), European Science Foundation, Oxford 1997. 
Ausbruch der Französischen Revolution (durch das Vorausdenken der Aufklärung), die Staatsrechtslehre nach 1871 (durch nachvollziehende Kommentierung) oder das Zustandekommen des Bürgerlichen Gesetzbuchs (durch Vorarbeit der Pandektistik) erklärt worden. In Wahrheit liegt in der Umschaffung des Sprachgebrauchs vor den »Ereignissen $"$ das eigentliche Ereignis, und umgekehrt erschafft erst die nachfolgende Deutung das Ereignis. Rechts- und Verfassungsgeschichte als Abfolge von sprachlich dokumentierten Bewußtseinszuständen einer Kommunikationsgemeinschaft derer, die an Recht und Verfassung beteiligt sind - diese Perspektive erleichtert jedenfalls die Erkennbarkeit feinerer Wechselwirkungen zwischen einem Sprechen, das zugleich Handeln ist, und einem Handeln, das nur in Form von Sprache »begriffen« und übermittelt werden kann.

Es geht also keineswegs darum, einen Primat des Denkens vor dem Handeln zu postulieren. Es geht um die Auflösung dieser Dichotomie. Neue Worte können, wenn sie einem breit empfundenen Bedürnfis entgegenkommen und Karriere machen, eine Veränderung des Koordinatensystems der Wahrnehmung bewirken. Das gleiche gilt für alte, längst in ihren Kontexten festgefahrene Worte, wenn sie in eine neue Beleuchtung oder einen neuen Kontext versetzt werden. Neue (oder alt-neue) Worte können schlagartig »aufklären « und desillusionieren. In Hans Christian Andersens genialem Text $»$ Des Kaisers neue Kleider« ereignet sich dies kraft des reinen Gemüts, das den konventionellen Kontext überschreitet und durch ein paar Worte den Funken der Aufklärung in die Menge wirft:

»Aber er hat ja gar nichts an!«, sagte endlich ein kleines Kind. »Herrgott, hört des Unschuldigen Stimme!«, sagte der Vater; und einer zischelte dem andern zu, was das Kind gesagt hatte. »Aber er hat ja gar nichts an! rief zuletzt das ganze Volk...«.

Die Beobachtung wirklichkeitsverändernder Benennung läßt sich historisch in jeder Epoche machen, soweit überhaupt $\mathrm{Zu}$ gang zu überlieferten Texten besteht. So kann man sich etwa der 
Formel »Staatsräson« widmen. Die italienischen Anfänge der "ragion di stato", die spanische, französische und englische Rezeptionsgeschichte, die deutsch-lateinische Umsetzung auf »ratio status « zeigen ein Kaleidoskop verschiedener Aneignungen, aber kein Chaos. Es handelt sich um ein Schlüsselwort Europas, dessen Karriere in sensibler Weise auf ganz unterschiedliche politische Situationen reagiert und gleichzeitig den großen Prozeß der Säkularisation und Verwissenschaftlichung des politischen Denkens widerspiegelt. »Ratio status « signalisiert einen neuen Ton des öffentlichen Sprechens, einen Kristallisationskern für staatszentriertes Denken. Man kann deutlich sehen, wie dieses Wortfeld dem Aufstieg des absolutistischen Fürstenstaats entweder »folgt«, ihn aber auch ideologisch »vorbereitet«. So kann einmal eine schon eingetretene Machtverschiebung nachfühlend begriffen, ins Bewußtsein gehoben, und damit eben »erschaffen « werden, in dem Sinn, in dem von der sprachlichen Konstruktion der Welt gesprochen werden kann. Daneben kann sich das Denken und Sprechen auch als einfache »Begleitung « darstellen. Was andere schon begriffen und formuliert haben, wird noch einmal ausgebreitet, verstärkt und populär gemacht - keine unwichtige Funktion, wenn man bedenkt, daß es nicht genügt, wenn ein einsames Genie einen Gedanken notiert. Die Veränderung der Welt durch Denken setzt eine massenhafte Verbreitung und eine allgemeine Durchdringung des öffentlichen Sprachgebrauchs voraus.

Als zweites Beispiel dient das Wort »Staat«. »Stato, état, state « und ihre zahlreichen Komposita setzen sich im Europa des 16. und 17. Jahrhunderts langsam durch, wandeln ihre Bedeutung von »Zustand « zu »Stand «, »Herrschaft«, »Gemeinwesen « und schließlich zu »hoheitlicher Bürokratie«. Im Wort »Statistik" steckt noch die Zustandsbeschreibung des Gemeinwesens, in den "Staatswissenschaften" das umfassende Wissen über Zustand, Herrschaft und Staat als Institution ${ }^{36}$.

36 P. L. Weinacht, Staat. Studien zur Bedeutungsgeschichte des Wortes von den Anfängen bis ins 19. Jahrhundert, Berlin 1968. 
Ein drittes Beispiel ist die »Policey« (Polizei). Die Geschichte ist bekannt: Aus der antiken »Politeia" (Politeuma) steigt am Ende des 15. Jahrhunderts, vermutlich im Umkreis des burgundischen Hofs, die Polluzey/Policey auf. Sie wird Synonymon für »Tätigkeit der Obrigkeit für die allgemeine Wohlfahrt» und verbreitet sich überall dort in der Frühen Neuzeit, wo eine Obrigkeit (government) das Leben der Bürger lenkt, reglementiert, wo ermahnt, befohlen und verboten wird ${ }^{37}$. Ein 1992 begonnenes Projekt der Quellenerschließung und -dokumentation der $\gg$ Policeyordnungen der Frühen Neuzeit« wird künftig eine Kartierung und Erschließung dieser massenhaften Normsetzung und-durchsetzung erlauben ${ }^{38}$.

Ziel der Policey ist - um ein weiteres Beispiel zu nennen die Wohlfahrt/welfare, Glückseligkeit/happiness. Auch dies sind Schlüsselworte der drei Jahrhunderte vor der Französischen Revolution $^{39}$. Aus mittelalterlichen Ursprüngen entfalten sie sich als Beschreibungen des »guten Lebensi, als Zielformel für das Handeln der Obrigkeiten. Sie changieren im 18. Jahrhundert stärker in Richtung auf das Individuum, bis schließlich die »Freiheit« die »Glückseligkeit« aufzehrt und vom Staat nur noch Sicherheit und Freiheit, aber kein »Glück« mehr verlangt ${ }^{40}$. Das »Glück « ist Sache des Individuums geworden: »Hans im Glück« definiert sein Glück selbst, töricht zwar nach dem Maßstab der Welt, aber souverän ${ }^{41}$.

37 H. Maier, Polizei, in: HRG, Bd. III (1984), Sp. 1800-1803; F.-L. Knemeyer, in: Geschichtliche Grundbegriffe Bd. 4, Stuttgart 1978, 875-897; K. Härter, Entwicklung und Funktion der Policeygesetzgebung des Heiligen Römischen Reiches Deutscher Nation im 16. Jahrhundert, in: Ius Commune XX (1993), 61-141.

38 K. Härter - M. Stolleis (Hrsg.), Repertorium der Policeyordnungen der Frühen Neuzeit, Bd.1: Deutsches Reich und geistliche Kurfürstentümer (Kurmainz, Kurköln, Kurtrier), hrsgg. v. K. Härter, Frankfurt a.M. 1996; vergleichend M. Stolleis (Hrsg.), Policey im Europa der Frühen Neuzeit, Frankfurt a.M. 1996.

39 M. Rassem, Wohlfahrt, in: Geschichtliche Grundbegriffe Bd.7, Stuttgart 1992, 595 636; M. Stolleis, Wohlfahrt, in: HRG, Bd. V (1995), Sp. 1486-1488.

40 D. Klippel, Freiheit und Freiheitsrechte im deutschen Naturrecht des 18. Jahrhunderts, Paderborn 1976.

41 Anders die kriminalpsychologische Deutung des Märchens bei $K$. Lüderssen, Hans im Glück (oben Anm. 2, 73 ff.). 
Nehmen wir abschließend noch drei Beispiele aus dem späten 18. und dem frühen 19. Jahrhundert: In den siebziger Jahren des 18. Jahrhunderts taucht in Deutschland das Wort »Selbstverwaltung" auf und durchläuft im 19. Jahrhundert eine große Laufbahn. Es wird zum zentralen Stichwort einer Gesellschaft, die sich staatsfrei »selbst verwalten « will, in den Gemeinden, in den Kirchen, im Handwerk und Gewerbe, schließlich in der Sozialversicherung ${ }^{42}$. Der von England faszinierte Verwaltungstheoretiker Rudolf von Gneist popularisierte schließlich das Wort »self-government ", das zwar schon in England verwendet, aber erst durch ihn zur Bezeichnung einer eigenen staatsunabhängigen Organisationsform wurde.

Oder das Wort »Staatsbürger«: Es tauchte im 18. Jahrhundert auf und wurde virulent politisch mit der französischen Revolution. Es signalisierte den Anspruch des Dritten Standes auf politische Mitbestimmung; denn Staatsbürger sollte der freie, am Staat Anteil nehmende »Bürger « sein, nicht mehr der subiectus, der »Untertan«. Populär wurde es durch Kant ${ }^{43}$. Wie politisch aufgeladen es war, sieht man daran, daß Regierungen des frühen 19. Jahrhunderts versuchten, seine Verwendung zu unterdrükken.

Schließlich das Wort »Rechtsstaat«. Wie das Wort »Staatsbürger« stammt es aus den letzten Jahren des 18. Jahrhunderts. Schriftlich belegt ist es erstmals für 1809 bei dem konservativen Romantiker Adam Müller. Aber die »kantische Schule« und die Liberalen bemächtigten sich seiner: Der Rechtsstaat sollte der Staat sein, der durch die von allen »Staatsbürgern « beschlossenen Gesetze, die volonté générale, gelenkt wird. Da alle an der Gesetzgebungsmacht Anteil haben, müssen sich alle unter das selbstgesetzte Recht beugen. »Rechtsstaat « wurde also eine liberale Parole gegen den spätabsolutistischen Staat, gegen Willkür

42 M. Stolleis, Selbstverwaltung, in: HRG, Bd. IV (1990), Sp. 1621-1625.

43 M. Stolleis, Untertan - Bürger - Staatsbürger, in: ders. Staat und Staatsräson in der frühen Neuzeit, Frankfurt a.M. 1990. 
und Unterdrückung von Freiheit. Die Geschichte seiner Formalisierung bzw. Re-Materialisierung ist die Geschichte des Kampfs um den Staatszweck, um das politische Leitbild dessen, was man vom Staat erwartete. Typischerweise tendierten Theoretiker, die einen Status quo zu verteidigen hatten, eher zum formalen Verständnis, während den interventionistisch gestimmten Vertretern einer Idee von »Gerechtigkeit « ein materialer Rechtsstaat normativ vorschwebte ${ }^{44}$.

Alle diese Beispiele kommen, wie man sieht, aus der Sphäre der Politik, der Staatstheorie und der Verfassungen. Es sind Kampf- und Tendenzbegriffe. Ihre Semantik ist voller Aggression, voller Interessen. Die Auseinandersetzungen um die Verwendung solcher Worte sind politischer Natur. Sie zielen auf Besetzung "positiver" Worte, sie suchen bestimmte Standpunkte durchzusetzen, indem sie die Schlüsselworte okkupieren - ein Vorgang, der in der Welt der politischen Parteien und der kommerziellen Werbung ganz geläufig ist ${ }^{45}$; denn die Besetzung eines Wortfeldes ist ein Stück Machtausübung.

Die Analyse der Wortverwendungen führt also mitten in die Geschichte - allerdings, um es zu wiederholen, nicht zur historischen Realität, sondern zur Ebene der in Sprache gefassten Gedanken. Was Historiker und Rechtshistoriker durch Sprachanalyse leisten können, ist Übersetzungsarbeit. Sie versuchen zu verstehen, wie ein Wort/Zeichen in einem uns fremd gewordenen Kontext verwendet wurde. Dazu bedarf es eines wenigstens partiellen sprachlichen Zugangs zum Sprachspiel, und es bedarf in der Regel mehrerer Quellen, damit Vergleiche überhaupt möglich sind. So war etwa, um ein ferner liegendes Beispiel zu wählen, die Entschlüsselung der Hieroglyphen nur möglich, weil die 1799 gefundene Inschrift auf dem Stein von Rosette endlich jenen partiellen Zugang bot, der es schließlich François Champollion ermöglichte, das Sprachsystem zu verstehen. Zwi-

44 M. Stolleis, Rechtsstaat, in: HRG, Bd. IV (1990), Sp. 367-375.

45 T. D. Weldon, The Vocabulary of Politics, London 1953. 
schen der Entschlüsselung einer unbekannten Schrift und derjenigen älterer Texte sehe ich keinen prinzipiellen Unterschied. Können auf diese Weise die Texte, wie man zu sagen pflegt, "zum Sprechen gebracht" werden, dann nur über das Medium unserer Sprache, unseres Weltverständnisses, unserer Meinungen und Vorurteile. Indem wir die Bedeutung eines Ausdrucks erklären, übersetzen wir ihn. Wir richten also den Blick auf vergangene Wirklichkeit, indem wir einen alten Text in einen neuen transformieren.

III.

Die Ergebnisse der bis hierher angestellten Überlegungen mögen banal erscheinen. Es scheint der Mühe nicht wert zu sein, zu erläutern, daß auch die »Begriffe $\ll$ nichts anderes sind als Worte, und daß hinter den »Tatsachen « nichts anderes steckt als sprachliche Botschaften, denen gegenwärtig aus pragmatischen Gründen allgemein Glauben geschenkt wird. Doch sind es nicht nur Akzentverschiebungen, die hierdurch bewirkt werden. Der Zweifel gegenüber dem »Begriff« bedeutet gerade für den Rechtshistoriker, daß er das Mißverständnis vermeiden kann, es gebe oberhalb der Quellentexte eine überhistorische sprachliche Ebene, der die sprachlichen Befunde zugeordnet werden könnten. Allzuleicht glauben Rechtshistoriker, die in Deutschland durchweg auch ausgebildete Juristen sind, sie könnten eben jene Figuren, die ihnen aus dem geltenden Recht geläufig sind, im älteren Material entdecken, ja eigentlich seien jene damaligen $\mathrm{Zu}-$ stände den heutigen in dem Sinne verwandt gewesen, daß vergleichbare Rechtsprobleme mit Hilfe der gleichen Begriffe gelöst worden seien. Die ehemals beliebte, heute schon verpönte Redeweise vom »Wesen « des Eigentums, des Besitzes, der Forderung, des Pfandes oder des Schuldverhältnisses signalisierte jene vermeintliche Ewigkeit von Figuren, an deren »Wiederkehr« man glaubte. Unzählige Dissertationen, deren Aufgabe es 
war, die Entwicklung eines heutigen Rechtsinstituts historisch nachzuzeichnen, haben sich, ausgerüstet mit dem Begriffsinstrumentarium des Bürgerlichen Gesetzbuchs, auf den Weg gemacht und in der Geschichte lauter »Vorformen " gefunden, in denen man entweder »noch « die Vorform oder »schon « das Gegenwärtige erblicken konnte. Einen verlässlichen Aussagewert über die Fragen, ob es wirklich die behaupteten Kontinuitäten gebe und ob die Funktion jener »Vorformen « denen der heutigen Institute vergleichbar war, ergaben diese Arbeiten nicht. So legitim und erhellend es ist, der Geschichte dogmatischer Figuren nach rückwärts nachzugehen, der Schluß vom Bekannten auf das Unbekannte, das sich manchmal sogar in einer »ähnlichen« Sprache maskiert, ist dann irreführend, wenn die älteren Texte nicht in ihrem Kontext, in ihrer Funktion für Familie und Haus, Wirtschaft und Politik als solche ernstgenommen, sondern zu Vorformen degradiert werden.

Ein weiterer wesentlicher Vorteil ergibt sich, wenn die $»$ Tatsache" als sprachliches Konstrukt und als Konvention des Fürwahrhaltens erkannt wird. Dieser Vorteil liegt in der Verunsicherung. Für sie gibt es Gründe, nämlich die genannten physiologischen und psychologischen Verzerrungen der Wahrnehmung, die Vermischung von Mythen und subjektiv eingefärbten Geschichtserzählungen sowie die massenhaften Fälschungen und Lügen, die zugleich als Dichtung geadelt sein können. Was die Sprachgemeinschaft nicht bezweifelt und was aus pragmatischen Gründen nicht lohnt, bezweifelt zu werden, mag immerhin »Tatsache« genannt werden, sofern die Möglichkeit erhalten bleibt, daß eines Tages eine bislang unbezweifelte »Tatsache" sich als Fiktion erweist oder eine neue Quelle eine bislang unbekannte »Tatsache« ans Licht fördert.

Nimmt man diese skeptische und relativistische Position ein, dann wird man auf der einen Seite sehr bescheiden, weil man begreift, wie wenig »zuverlässig« zu erfahren ist und wie viele Fallgruben es gibt, in die man fällt, wenn man einen Text für bare Münze nimmt. Gleichzeitig wird man aber methodisch an- 
spruchsvoller, weil sich nun auch die Vision einer sprachlich vermittelten »histoire totale» einstellt, also einer Geschichtsschreibung, die simultan auf mehreren Ebenen arbeitet, die die Daten der Sozial- und Wirtschaftsgeschichte, der Politik- und Ereignisgeschichte ebenso integriert wie die Rechtsgeschichte, die Geschichte der Moral, der Kultur und der Sitten. Wird die Menschenwelt in der sprachlichen Kommunikation »begriffen«, "gedeutet" und auf diese Weise »erschaffen", so daß sich Menschen in ihr orientieren und verständigen können, dann umfasst die Welt der Zeichen auch alle Lebensvorgänge. In ihr ist die Analyse der menschlichen Theoriebildung durch »Worte« und »Begriffe" sowie der geistigen und praktischen Organisation des Wissens - vulgo Wissenschaftsgeschichte - nur ein Teilgebiet, allerdings ein wichtiges und der Anstrengung des Historikers würdiges. 


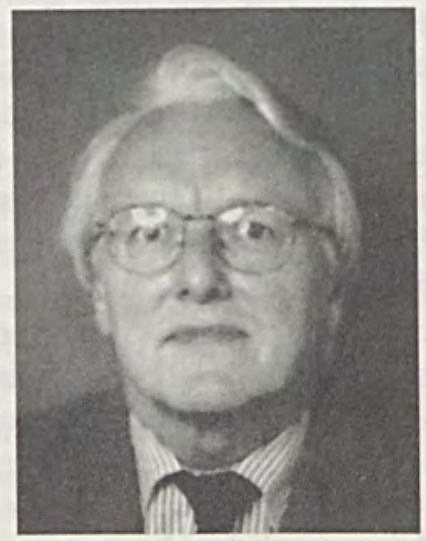

\section{Michael Stolleis}

1941

1961-1965

1967

1973

1974

1991

1991

1995 geb. in Ludwigshafen/Rh.

Studium der Rechtswissenschaft in Heidelberg, Würzburg und München, 1. u. 2. Staatsexamen 1965/1969

Promotion in München (Sten Gagnér)

Habilitation für Öffentliches Recht, Neuere Rechtsgeschichte und Kirchenrecht in München

Berufung an die Universität Frankfurt a.M.

Leibniz-Preis der Deutschen Forschungsgemeinschaft

Direktor am Max-Planck-Institut für Europäische Rechtsgeschichte Mitglied der Akademien in Mainz, Göttingen und Berlin sowie der Wiss. Gesellschaften Frankfurt a.M. und Lund/Schweden.

Research Award des Riksbanken Jubileums Fonds Stockholm

\section{Bücher u.a.:}

Staatsräson, Recht und Moral in philosophischen Texten des späten 18. Jahrhunderts, 1972; Gemeinwohlformeln im nationalsozialistischen Recht, 1974; Quellen zur Geschichte des Sozialrechts, 1976; Geschichte des öffentlichen Rechts in Deutschland, Bd. 1 (1988), 2 (1992), 3 (i. Vorber.); Staat und Staatsräson in der frühen Neuzeit, 1990; Recht im Unrecht. Studien zur Rechtsgeschichte des Nationalsozialismus, Frankfurt 1994 (engl. Chicago 1997); (Hrsg.), Staatsdenker der frühen Neuzeit, 3. Aufl. 1995; (Hrsg.), Juristen. Ein biographisches Lexikon, 1995. 


\section{Würzburger Vorträge zur Rechtsphilosophie, Rechtstheorie und Rechtssoziologie}

Heft 1: Arthur Kaufmann: Theorie der Gerechtigkeit. Problemgeschichtliche Betrachtungen, 1984, 51 S., 19,80 DM

Heft 3: Niklas Luhmann: Die soziologische Beobachtung des Rechts, 1986, 48 S., 16,80 DM

Heft 4: Ernst-Wolfgang Böckenförde: Die verfassunggebende Gewalt des Volkes - Ein Grenzbegriff des Verfassungsrechts, 1986, 34 S., $16,80 \mathrm{DM}$

Heft 5: Ralf Dreier: Rechtsbegriff und Rechtsidee. Kants Rechtsbegriff und seine Bedeutung für die gegenwärtige Diskussion, 1986, 37 S., $18,80 \mathrm{DM}$

Heft 6: Günter Dux: Der Täter hinter dem Tun. Zur soziologischen Kritik der Schuld, 1988, 58 S., 24,--DM

Heft 7: Franz Bydlinski: Recht, Methode und Jurisprudenz, 1987, 46 S., $19,80 \mathrm{DM}$

Heft 8: Martin Kriele: Freiheit und „Befreiung“. Gibt es eine Rangordnung v der Menschenrechte?, 1988. 52 S., 26,-DM

Heft 9: Manfred Rehbinder: Fortschritte und Entwicklungstendenzen einer Soziologie der Justiz, 1989, 63 S., 26,- DM

Heft 10: Klaus Lüderssen: Die Krise des öffentlichen Strafanspruchs, 1989, 62 S., 26,-DM

Heft 11: Norbert Hoerster: Verteidigung des Rechtspositivismus, 1989, 31 S., 26,-DM

Heft 12: Guiseppe Duso: Der Begriff der Repräsentation bei Hegel und das moderne Problem der politischen Einheit, 1990, 55 S., 24,- DM

Heft 13: Otfried Höffe: Gerechtigkeit als Tausch? Zum politischen Projekt der Moderne, 1991, 37 S., 18,- DM

Heft 14: Klaus F. Röhl: Die Gerechtigkeitstheorie des Aristoteles aus der Sicht sozialpsychologischer Gerechtigkeitsforschung, 1992, 59 S., 26,- DM 


\section{Würzburger Vorträge zur Rechtsphilosophie, Rechtstheorie und Rechtssoziologie}

Heft 15: Hans Albert: Rechtswissenschaft als Realwissenschaft. Das Recht als soziale Tatsache und die Aufgabe der Jurisprudenz, 1993, 37 S., 19,- DM

Heft 16: Gerd Irrlitz: Moral und Methode. Die Struktur in Kants Moralphilosophie und die Diskursethik, 1994, 56 S., 26,-DM

Heft 17: Hasso Hofmann: Gebot, Vertrag, Sitte. Die Urformen der Begründung von Rechtsverbindlichkeit, 1993, 49 S., 26,- DM

Heft 18: Klaus Adomeit: Das bürgerliche Recht, das Bürgerliche Gesetzbuch und die bürgerliche Gesellschaft, 1996, 42 S., 26,-DM

Heft 19: Wclgang Schild: Schuld und Unfreiheit. Gedanken zur Strafjustiz und Psychoanalyse in Leonhard Franks »Die Ursache«, 1996, 50 S., 26,- DM

Heft 20: Kurt Seelmann: Theologie und Jurisprudenz an der Schwelle zur Moderne. Die Geburt des neuzeitlichen Naturrechts in der iberischen Spätscholastik. 1997, 36 S., 22,- DM

Heft 21: Winfried Brugger: Menschenwürde, Menschenrechte, Grundrechte. 1997, 54 S., brosch., 28,- DM 